Recent Insights into the Physics of the Sun and Heliosphere:

Highlights from SOHO and Other Space Missions

IAU Symposium, Vol. 203, 2001

P. Brekke, B. Fleck, and J. B. Gurman eds.

\title{
Velocities in Sunspot Plumes
}

\author{
P. Maltby, O. Kjeldseth-Moe and N. Brynildsen \\ Institute of Theoretical Astrophysics, University of Oslo, P.O. Box \\ 1029, 0315 Oslo, Norway
}

\begin{abstract}
We have extended our investigation of sunspot regions, with particular attention to the physics of the transition region between the chromosphere and the corona. In sunspot plumes, the most prominent features in the transition region intensity maps, we find downflows exceeding $25 \mathrm{~km} \mathrm{~s}^{-1}$. The observations show that the flow cannot be maintained by inflow from the corona and prompt a search for the origin of the flow and its possible relation to the origin of the sunspot plume. We find that the downflows in sunspot plumes are maintained by gas at transition region temperatures, streaming in flow channels from locations well outside the sunspot. Hence, the flow pattern in the transition region shows similarities to the inverse Evershed flow in the chromosphere. We present a working hypothesis that is based on the siphon flow mechanism.
\end{abstract}

\section{Introduction}

Sunspot plumes, detected by Foukal et al. (1974), are the most prominent spectral line emission features above sunspots in the transition region between the chromosphere and the corona. In this paper the focus is on the downflow in sunspot plumes. We search for the origin of the sunspot plume and its downflow by studying the spatial distribution of line emission and line-of-sight velocity in the chromosphere, transition region, and corona. This investigation is based on simultaneous observations of ten or six EUV emission lines in 50 sunspot regions with the Coronal Diagnostic Spectrometer - CDS (Harrison et al. 1995) on the Solar and Heliospheric Observatory - SOHO. Nineteen of the sunspots are observed on two or more days during their passage over the disk, five sunspots are studied as they cross the solar limb.

\section{Summary}

1. The observations show that 37 out of 50 sunspot regions contain one or two sunspot plumes in the Ne VI $\lambda 562$ line, 32 of the sunspots show plumes in the $\mathrm{O} \vee \lambda 629$ line. The corresponding numbers for the $\mathrm{O}$ IV $\lambda 554$ and $\mathrm{O}$ III $\lambda 599$ lines are 24 and 4, respectively. There are no plumes in the $\mathrm{Mg}$ IX $\lambda 368$ line. Hence, the visibility of sunspot plumes changes with the line formation temperature within the transition region. 